\title{
First-principles study of structural, electronic and elastic properties of $\mathrm{Nb}_{4} \mathrm{AlC}_{3}$
}

\author{
A. Bouhemadou* \\ Laboratory for Developing New Materials and their Characterization, \\ Department of Physics, Faculty of Science, University of Setif, 19000 Setif, Algeria. and \\ Department of Physics and Astronomy, College of Science, \\ King Saud University, P.O. Box 2455, Riyadh 11451, Saudi Arabia.
}

(Received on 23 October, 2009)

\begin{abstract}
Using First-principles calculations, we have studied the structural, electronic and elastic properties of $\mathrm{Nb}_{4} \mathrm{AlC}_{3}$, a new compound belonging to the MAX phases. Geometrical optimization of the unit cell is in good agreement with the experimental data. The effect of high pressures, up to $20 \mathrm{GPa}$, on the lattice constants shows that the contractions are higher along the $c$-axis than along the $a$-axis. We have observed a quadratic dependence of the lattice parameters versus the applied pressure. The band structure shows that this compound is electrical conductor. The analysis of the site and momentum projected densities shows that bonding is due to $\mathrm{Nb} d-\mathrm{C} p$ and $\mathrm{Nb} d-\mathrm{Al} p$ hybridizations. The $\mathrm{Nb} d$-C $p$ bond is lower in energy and stiffer than $\mathrm{Nb} d$ - $\mathrm{Al}$ $p$ bond. The elastic constants are calculated using the static finite strain technique. We derived the bulk and shear moduli, Young's modulus and Poisson's ratio for ideal polycrystalline $\mathrm{Nb}_{4} \mathrm{AlC}_{3}$ aggregate. We estimated the Debye temperature of $\mathrm{Nb}_{4} \mathrm{AlC}_{3}$ from the average sound velocity. This is the first quantitative theoretical prediction of the elastic properties of $\mathrm{Nb}_{4} \mathrm{AlC}_{3}$ compound, and it still awaits experimental confirmation.
\end{abstract}

Keywords: MAX phases; Ab initio; Structural properties; Electronic properties; Elastic properties; Pressure effect

\section{INTRODUCTION}

The $\mathrm{M}_{n+1} \mathrm{AX}_{n}$, also called the MAX phases, where $\mathrm{M}$ is an early transition metal, $\mathrm{A}$ is an $\mathrm{A}$ group element, $\mathrm{X}$ is either $\mathrm{C}$ or $\mathrm{N}$ and $\mathrm{n}=1-3$, attract more and more attention due to the combination of properties usually associated with metals and ceramics (for details see Ref. 1 and the references cited therein). Like metals, they are electrically and thermally conductive, not susceptible to thermal shock, plastic at high temperature and exceptionally damage tolerant, and most readily machinable. Like ceramics, they are elastically rigid, lightweight, creep and fatigue resistant and maintain their strengths to high temperatures [2-12]. This makes them attractive for many applications such as structural materials at elevated temperature. More than 50 compounds were reported for $\mathrm{n}=1$ (the so-called 211 phases). For compounds with $\mathrm{n}=2$, four members (the so-called 312 phases), were identified. When $\mathrm{n}$ is increased to 3 , (the so-called 413 phases), four compounds were synthesized [13-16].

Recently $\mathrm{Hu}$ et al [16] reported that $\mathrm{Nb}_{4} \mathrm{AlC}_{3}$, a new compound belonging to the MAX phases, was discovered by annealing bulk $\mathrm{Nb}_{2} \mathrm{AlC}$ at $1700^{\circ} \mathrm{C}$. The crystal structure of $\mathrm{Nb}_{4} \mathrm{AlC}_{3}$ was determined by combined $\mathrm{X}$-ray diffraction and high-resolution transmission electron microscopy. It was reported that $\mathrm{Nb}_{4} \mathrm{AlC}_{3}$ follows the $\mathrm{Ti}_{4} \mathrm{AlN}_{3}$-type crystal structure [17]. Its unit cell contains two formula units, with space group P63/mmc (\#194). The atomic positions are $\mathrm{Nb} 1$ at $4 \mathrm{f}$ $\left(1 / 3,2 / 3, Z_{N b 1}\right), \mathrm{Nb} 2$ at $4 \mathrm{e}\left(0,0, Z_{N b 2}\right), \mathrm{Al}$ at $2 \mathrm{c}(1 / 3,2 / 3$, $1 / 4), \mathrm{C} 1$ at $2 \mathrm{a}(0,0,0)$ and $\mathrm{C} 2$ at $4 \mathrm{f}\left(1 / 3,2 / 3, Z_{C}\right)$, where $Z_{N b 1}, Z_{N b 2}$ and $Z_{C}$ are the internal free coordinates. The structure is thus defined by two lattice parameters, $a$ and $c$, and the three internal structural parameters, $\mathrm{ZNb}_{1}, \mathrm{ZNb}_{2}$ and $Z_{C}$. Fig. 1 shows the unit cell of $\mathrm{Nb}_{4} \mathrm{AlC}_{3}$.

In order to fully take advantage of the properties of $\mathrm{Nb}_{4} \mathrm{AlC}_{3}$ for eventual technological applications, a theoreti-

${ }^{*}$ Electronic address: a_bouhemadou@yahoo.fr

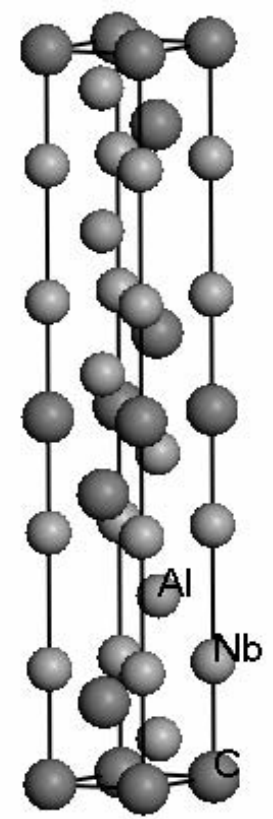

FIG. 1: The unit cell of $\mathrm{Nb}_{4} \mathrm{AlC}_{3}$.

cal investigation of the structural, elastic and electronic properties is necessary. First-principles calculations offer one of the most powerful tools for carrying out theoretical studies of these properties. In the present work, we report first principles study of structural, elastic and electronic properties for $\mathrm{Nb}_{4} \mathrm{AlC}_{3}$ compound, using the state of the art pseudopotential plane wave method (PP-PW), in the framework of the density functional theory (DFT) within the local density approximation (LDA).

The paper is organized as follows: In section 2, we briefly describe the computational techniques used in this work. Results and discussions of the structural, electronic and elastic properties are presented in section 3. Finally, conclusions and remarks are given in section 4. 


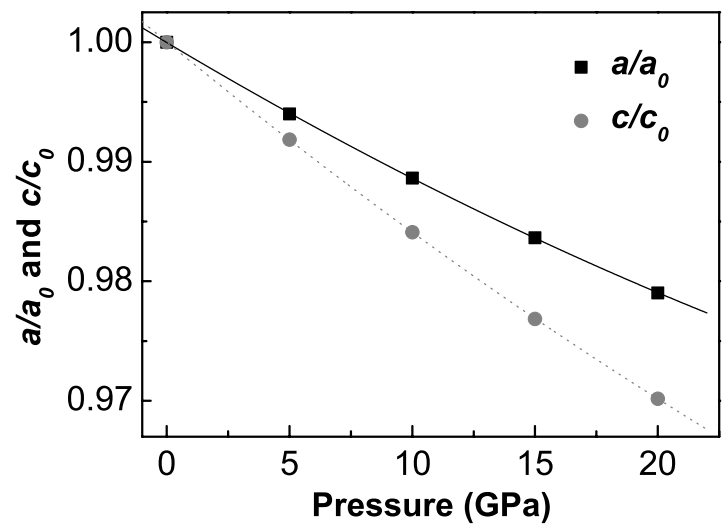

FIG. 2: Pressure dependence of the relative lattice parameters $\left(a / a_{0}\right.$ and $c / c_{0}$ ) for $\mathrm{Nb}_{4} \mathrm{AlC}_{3}$ compound. The solid lines are least-square fits of the data points to a quadratic polynomial.

\section{COMPUTATIONAL METHODS}

The first-principles calculations were performed by employing pseudo-potential plane- waves (PP-PW) approach based on the density functional theory (DFT) $[18,19]$ and implemented in the CASTEP code [20]. The major advantages of this approach are: the ease of computing forces and stresses; good convergence control with respect to all employed computational parameters; favourable scaling with number of atoms in the system and the ability to make cheaper calculations by neglecting core electrons. The exchange-correlation potential is treated within the LDA, developed by Ceperly and Alder and parameterized by Perdew and Zunger [21,22]. The presence of tightly-bound core electrons was represented by non local ultra-soft pseudopotentials of the Vanderbilt-type [23]. The states $C 2 s^{2} 2 p^{2}$, Al $3 s^{2} 3 p^{1}$ and $\mathrm{Nb} 4 s^{2} 4 p^{6} 4 d^{4} 5 s^{1}$ were treated as valence states. The two parameters that affect the accuracy of calculations are the kinetic energy cut-off which determines the number of plane waves in the expansion and the number of special k-points used for the Brillouin zone (BZ) integration. We performed convergence with respect to BZ sampling and the size of the basis set. Converged results were achieved with $9 \times 9 \times 2$ special k-points mesh [24]. The size of the basis set is given by cut-off energy equal to $360 \mathrm{eV}$. Careful convergence tests show that with these parameters relative energy converged to better than $5 \times 10-6 \mathrm{eV} /$ atom.

\section{RESULTS AND DISCUSSION}

\subsection{Structural properties}

The structural parameters of $\mathrm{Nb}_{4} \mathrm{AlC}_{3}$ were determined using the Broyden-Fletcher-Goldfarb-Shenno (BFGS) minimization technique, with the flowing thresholds for converged structures: energy change per atom less than $5 \times 10$ $6 \mathrm{eV}$, residual force less than $0.01 \mathrm{eV} / \AA$, and the displacement of atoms during the geometry optimization less than 0.0005 $\AA$.

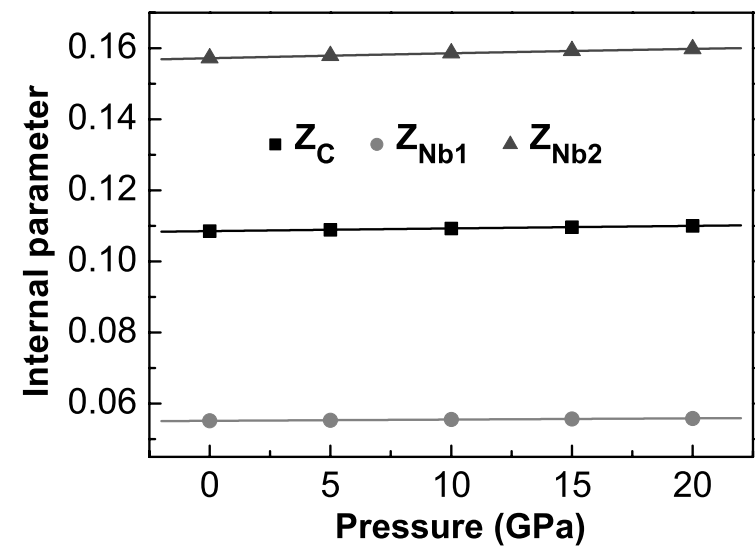

FIG. 3: Pressure dependence of the internal structural parameters $\left(\mathrm{Z}_{N b 1}, \mathrm{Z}_{N b 2}\right.$ and $\left.\mathrm{Z}_{C}\right)$ for $\mathrm{Nb}_{4} \mathrm{AlC}_{3}$ compound. The solid lines are least-square fits of the data points to a quadratic polynomial.

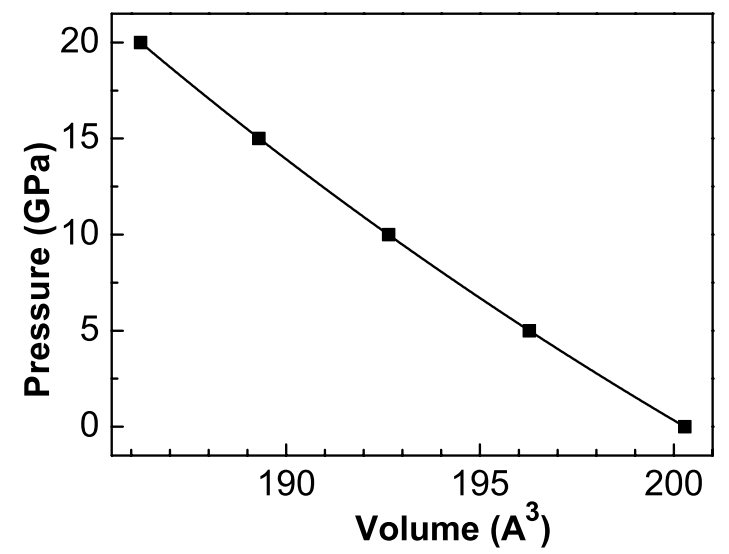

FIG. 4: The calculated pressure-volume relations for $\mathrm{Nb}_{4} \mathrm{AlC}_{3}$ compound. The solid lines are given by the Birch-Murnaghan equation of state with the parameters listed in Table 1 .

The calculated lattice parameters, $a_{0}$ and $c_{0}$, and the internal structural parameters $\mathrm{Z}_{N b 1}, \mathrm{Z}_{N b 2}$ and $\mathrm{Z}_{C}$, for $\mathrm{Nb}_{4} \mathrm{AlC}_{3}$, as determined from geometry at $\mathrm{P}=0 \mathrm{GPa}$, are given in $\mathrm{Ta}$ ble 1 together with the available experimental and theoretical data. There is a good agreement between our results and the experimental ones [16]. The computed lattice constants $a$ and $c$ for $\mathrm{Nb}_{4} \mathrm{AlC}_{3}$ deviate from the measured ones within 0.46 and $0.57 \%$, respectively. The deviation from the experimental value of $c / a$ for $\mathrm{Nb}_{4} \mathrm{AlC}_{3}$ is found to be $0.29 \%$. This ensures the reliability of the present first-principles computations.

In order to show how the structural parameters in this compound behave under pressure, the equilibrium geometries of $\mathrm{Nb}_{4} \mathrm{AlC}_{3}$ unit cells were computed at fixed values of applied hydrostatic pressure in the 0 to $20 \mathrm{GPa}$ range with the step of $5 \mathrm{GPa}$, where, at each pressure, a complete optimization for the structural parameters was performed. In Figs. 2 and 3, we plot the variation of the relative changes of the lattice parameters $\left(a / a_{0}\right.$ and $\left.c / c_{0}\right)$ and the internal parameters $\left(\mathrm{Z}_{N b 1}\right.$, $\mathrm{Z}_{N b 2}$ and $\mathrm{Z}_{C}$ ) versus applied hydrostatic pressure $(p)$. We ob- 
TABLE 1: Structural parameters of $\mathrm{Nb}_{4} \mathrm{AlC}_{3}$ at zero-pressure: lattice constants, $a_{0}$ and $c_{0}$, internal parameters, $\mathrm{Z}_{N b 1}, \mathrm{Z}_{N b 2}$ and $\mathrm{Z}_{C}$, bulk modulus, $B_{0}$, and its pressure derivative, $B^{\prime}$; along with the available measured results. The values of $B_{0}$ and $B^{\prime}$ are evaluated from the fitting of the pressure-volume data to a third-order Birch-Murnaghan equation of state; equation (1) in the text.

\begin{tabular}{lllllllll}
\hline & $a_{0}(\AA)$ & $c_{0}(\AA)$ & $c_{0} / a_{0}$ & $B_{0}(\mathrm{GPa})$ & $B^{\prime}$ & $\mathrm{Z}_{\mathrm{Nb} 1}$ & $\mathrm{Z}_{\mathrm{Nb} 21}$ & $\mathrm{Z}_{\mathrm{C}}$ \\
\hline This work & 3.1053 & 23.9827 & 7.7231 & 237 & 4.07 & 0.05514 & 0.157187 & 0.108531 \\
Exp. [16] & 3.1296 & 24.1208 & 7.7073 & - & - & 0.0553 & 0.1574 & 0.1086 \\
\hline
\end{tabular}

TABLE 2: The calculated first- $\left(\alpha\right.$ in $\left.10^{-4} \mathrm{GPa}^{-1}\right)$ and second-order $\left(\beta\right.$ in $\left.10^{-5} \mathrm{GPa}^{-2}\right)$ pressure coefficients of the relative lattice parameters $\left(a / a_{0}, c / c_{0}\right)$ and internal structural parameters $\left(\mathrm{Z}_{N b 1}, \mathrm{Z}_{N b 2}\right.$ and $\left.\mathrm{Z}_{C}\right)$ for $\mathrm{Nb}_{4} \mathrm{AlC}_{3}$.

\begin{tabular}{cccccc}
\hline & $a / a_{0}$ & $c / c_{0}$ & $\mathrm{Z}_{\mathrm{Nb} 1}$ & $\mathrm{Z}_{\mathrm{Nb} 2}$ & $\mathrm{Z}_{\mathrm{C}}$ \\
\hline & & & & & \\
\hline & -12.30 & -16.90 & 0.3603 & 1.4812 & 0.7841 \\
$\beta$ & 0.8964 & 0.9922 & -0.0051 & -0.0843 & -0.0226 \\
\hline
\end{tabular}

TABLE 3: The calculated elastic constants, $C_{i j}$ (in GPa) for $\mathrm{Nb}_{4} \mathrm{AlC}_{3}$.

\begin{tabular}{lllllll}
\hline$C_{11}$ & $C_{33}$ & $C_{44}$ & $C_{12}$ & $C_{13}$ & $C_{66}$ \\
\hline & & & & & \\
445 & 349 & 176 & 143 & 152 & 151 \\
\hline
\end{tabular}

TABLE 4: The calculated bulk moduli (in GPa), $B_{V}$ (Voigt's bulk modulus), $B_{R}$ (Reuss's bulk modulus) and $B=\left(B_{R}+B_{V}\right) / 2$, shear moduli (in GPa), $G_{R}$ (Reuss's shear modulus), $G_{V}$ (Voigt's shear modulus) and $G=\left(G_{R}+G_{V}\right) / 2$, Young's modulus (in GPa), $E$, and Poisson's ratio, $v$, for $\mathrm{Nb}_{4} \mathrm{AlC}_{3}$.

\begin{tabular}{cccccccc}
\hline$B_{R}$ & $B_{V}$ & $B$ & $G_{R}$ & $G_{V}$ & $G$ & $E$ & $v$ \\
\hline 234.5 & 237.0 & 235 & 149.0 & 153.4 & 151 & 374 & 0.2358 \\
\hline
\end{tabular}

serve a quadratic dependence in the considered range of pressure. The same behaviour has been previously observed for $\mathrm{Ta}_{4} \mathrm{AlC}_{3}$ [14]. The solid curve is the quadratic least-squares fit. The values of the linear and quadratic pressure coefficients of $a / a_{0}, c / c_{0}, \mathrm{Z}_{N b 1}, \mathrm{Z}_{N b 2}$ and $\mathrm{Z}_{C}$ for $\mathrm{Nb}_{4} \mathrm{AlC}_{3}$ are given in Table 2. Fig. 2 shows that the compressibility of
TABLE 5: The calculated density ( $\rho$ in $\mathrm{g} / \mathrm{cm} 3)$, the longitudinal, transverse and average sound velocity $\left(\mathrm{v}_{l}, \mathrm{v}_{t}, \mathrm{v}_{m}\right.$ in $\left.\mathrm{m} / \mathrm{s}\right)$ calculated from polycrystalline elastic modulus, and the Debye temperatures $\left(\theta_{D}\right.$ in $\left.\mathrm{K}\right)$ derived from the average sound velocity for $\mathrm{Nb}_{4} \mathrm{AlC}_{3}$.

\begin{tabular}{lllll}
\hline$\rho$ & $v_{l}$ & $v_{t}$ & $v_{m}$ & $\theta_{D}$ \\
& & & & \\
\hline 7.21 & 7791 & 4581 & 5077 & 517 \\
\hline
\end{tabular}

$\mathrm{Nb}_{4} \mathrm{AlC}_{3}$ is greater along the $c$ axis than along the $a$ axis.

The calculated unit cell volumes at fixed values of applied hydrostatic pressure in the 0 to $20 \mathrm{GPa}$ range with the step of $5 \mathrm{GPa}$ were used to construct the equation of state (EOS), which was fitted to a third-order Birch-Murnaghan equation [25] (Fig. 4):

$$
P=\frac{3}{2} B_{0}\left[\left(\frac{V_{0}}{V}\right)^{\frac{7}{3}}-\left(\frac{V_{0}}{V}\right)^{\frac{5}{3}}\right]\left[1+\frac{3}{4}\left(4-B^{\prime}\right)\left\{\left(\frac{V_{0}}{V}\right)^{\frac{2}{3}}-1\right\}\right]
$$

Here $V_{0}$ is fixed at the value determined from the zeropressure data. We obtained, by least-squares fit, the bulk modulus $B_{0}$ at zero pressure, which represents the resistance to volume change and is related to the overall atomic binding properties in material, and its pressure derivative $B$ '. These are listed in Table 1.

\subsection{Electronic properties}

The calculated energy band structure for $\mathrm{Nb}_{4} \mathrm{AlC}_{3}$, at equilibrium lattice parameters, along the high symmetry directions in the Brillouin zone is shown in Fig. 5. The valence and conduction bands overlap considerably and there is no band gap at the Fermi level. As a result $\mathrm{Nb}_{4} \mathrm{AlC}_{3}$ will exhibit metallic properties.

To further elucidate the nature of chemical bonding in this compound, we study the partial density of states (PDOS) of $\mathrm{Nb}_{4} \mathrm{AlC}_{3}$ as shown in Fig. 6. An important feature is the existence of a pseudo gap in the DOS in the vicinity of the Fermi level, which is an indication for stability [26]. Car- 


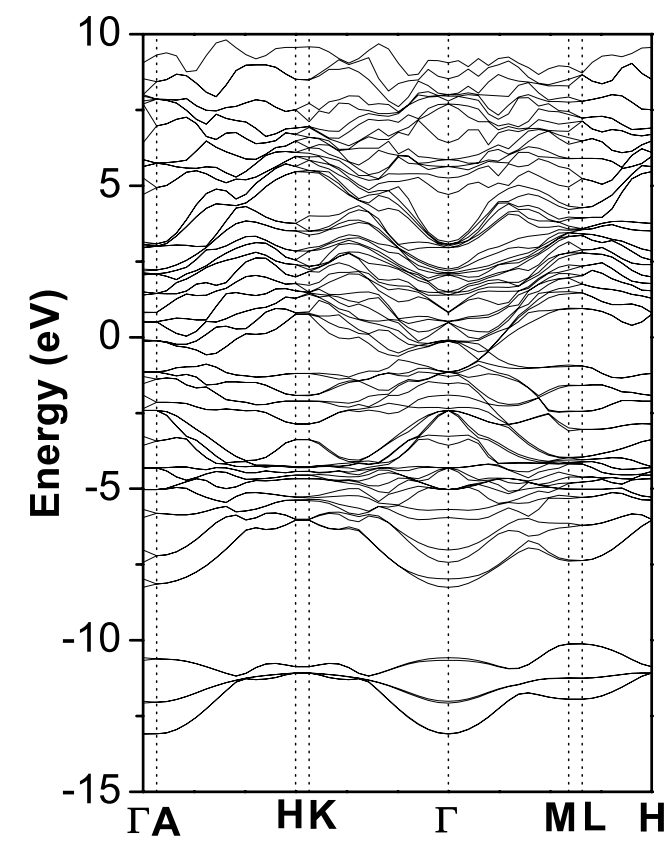

FIG. 5: Band structures along the principal high-symmetry directions in the Brillouin zone of $\mathrm{Nb}_{4} \mathrm{AlC}_{3}$ compound.

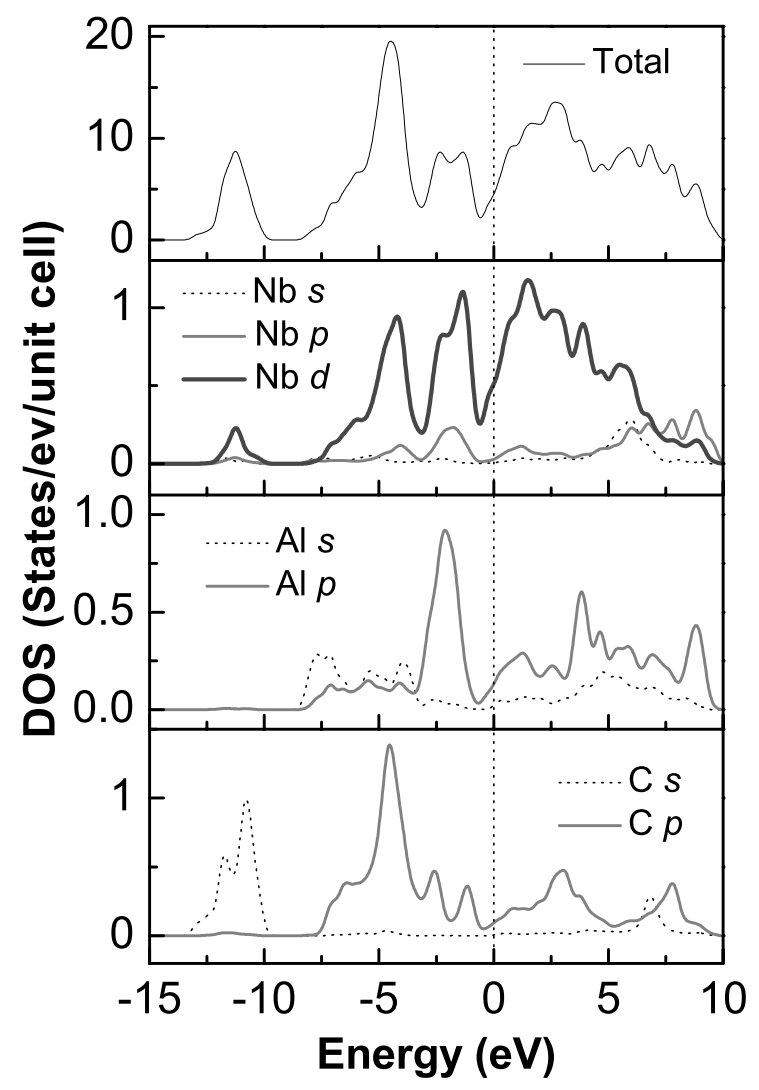

FIG. 6: Partial density of states (PDOS) for $\mathrm{Nb}_{4} \mathrm{AlC}_{3}$ compound as a function of energy. Fermi level is aligned with $0 \mathrm{eV}$. bon does not contribute to the DOS at the Fermi level and therefore is not involved in the conduction properties. $\mathrm{Nb}$ $d$ electrons are mainly contributing to the DOS at the Fermi level, and should be involved in the conduction properties. $\mathrm{Al}$ electrons do not contribute significantly at the Fermi level due to a scooping effect resulting from the presence of the $\mathrm{Nb} d$ states. These results are consistent with previous reports on MAX phases [27]. It is apparent that a covalent interaction occurs between the constituting elements due to the fact that states are degenerate with respect to both angular momentum and lattice site. $\mathrm{C} p$ and $\mathrm{Nb} d$ as well as $\mathrm{Al} p$ and $\mathrm{Nb} d$ states are hybridized. Also, due to the difference in the electronegativity between the comprising elements, some ionic character can be expected. The bonding character may be described as a mixture of covalent-ionic and, due to the $d$ resonance in the vicinity of the Fermi level, metallic. The pseudo gap is likely to split the bonding and anti bonding orbitals. The PDOS shows an other interesting feature: the hybridization peak of $\mathrm{Nb} d$ and $\mathrm{C} p$ lies lower in energy than that of $\mathrm{Nb} d$ and $\mathrm{Al} p$. This suggests that the $\mathrm{Nb} d-\mathrm{C} p$ bond is stiffer than the $\mathrm{Nb} d-\mathrm{Al} p$ bond.

\subsection{Elastic properties}

It is well established that first principles studies based on DFT can be used to obtain reliable elastic properties of inorganic compounds [28]. Several methods are available for computation of stiffness coefficients, but currently the finite strain method, used in the present work, seems to be the most commonly used one. In this approach, the ground state structure is strained according to symmetry-dependent strain patterns with varying amplitudes and a subsequent computing of the stress tensor after a re-optimization of the internal structure parameters, i.e. after a geometry optimization with fixed cell parameters. The elastic stiffness coefficients are then the proportionality coefficients relating the applied strain to the computed stress. Both stress and strain have three tensile and three shear components, giving six components in total. The linear elastic stiffnesses, $C i j$, form thus a $6 \times 6$ symmetric matrix with a maximum of 21 different components, such that $\sigma_{i}=C_{i j} \varepsilon_{j}$ for small stresses $s$, and strains, $e$ [29,30]. Any symmetry present in the structure may make some of these components equal and may let other components vanish. A hexagonal crystal has six different symmetry elements $\left(C_{11}\right.$, $C_{12}, C_{13}, C_{33}, C_{44}$, and $C_{66}$ ), and only five of them are independent since $C_{66}=\left(C_{11}-C_{12}\right) / 2$. For the hexagonal system, two different strain patterns, one with non-zero first and fourth components, and another with a non-zero third component, give stresses that are related to all five independent elastic coefficients [31-33]. Three positive and three negative amplitudes were used for each strain component with the maximum value of $0.5 \%$. The elastic stiffness coefficients were then determined from a linear fit of the calculated stress as a function of strain.

In Table 3, we list the calculated values of the elastic constants $C_{i j}$. We are not aware of any experimental data on the elastic properties. Future experimental measurements will test our calculated predictions.

Once the elastic constants are determined, we would like 
to compare our results with experiments, or predict what an experiment would yield for the elastic constants. A problem arises when single crystal samples cannot be obtained. Then it is not possible to measure the individual elastic constants $C_{i j}$. Instead, the isotropic bulk modulus $B$ and shear modulus $G$ are determined [34]. These quantities cannot, in general, be calculated directly from the $C_{i j}$, but we can use our values to place bounds on the isotropic moduli. Reuss found lower bounds, the Reuss bulk modulus $\left(B_{R}\right)$ and the Reuss shear modulus $\left(G_{R}\right)$, for all lattices [34,35], while Voigt discovered upper bounds, the Voigt bulk modulus $\left(B_{V}\right)$ and the Voigt shear modulus $\left(G_{V}\right)$ [34,36]. Hill has shown that the Voigt and Reuss averages are limits and suggested that the actual effective moduli could be approximated by the arithmetic mean of the two bounds [37]. We also calculated the Young's modulus, E, and Poisson's ratio, v, which are frequently measured for polycrystalline materials when investigating their hardness. These quantities are related to the bulk modulus $B$ and the shear modulus $G$ by the following equations [34]:

$$
\begin{aligned}
& E=9 B G /(3 B+G) \\
& \nu=(3 B-E) /(6 B)
\end{aligned}
$$

The calculated bulk modulus $B_{0}$, shear modulus $G$, Young's modulus $E$ and Poisson's ratio $v$ of $\mathrm{Nb}_{4} \mathrm{AlC}_{3}$ are given in Table 4. From Tables 1 and 4 , we can see that the calculated value of $B_{V}$, one limiting of the bulk modulus, from the elastic constants (Table 4) has nearly the same value as the one obtained from the EOS fitting (Table 1). This might be an estimate of the reliability and accuracy of our calculated elastic constants for $\mathrm{Nb}_{4} \mathrm{AlC}_{3}$ compound.

\subsection{Calculation of Debye temperature}

Having calculated the Young's modulus E, bulk modulus $B$, and shear modulus $G$, one can calculate the Debye temperature, which is an important fundamental parameter closely related to many physical properties such as elastic constants, specific heat and melting temperature.

At low temperature the vibrational excitation arises solely from acoustic modes. Hence, at low temperatures the Debye temperature calculated from elastic constants is the same as that determined from specific heat measurements. One of the standard methods to calculate the Debye temperature $\left(\theta_{D}\right)$ is from elastic constants data, since $\theta_{D}$ may be estimated from the average sound velocity, $v_{m}$ by the following equation $[38,39]$ :

$$
\theta_{D}=\frac{h}{k_{B}}\left[\frac{3}{4 \pi V_{a}}\right]^{1 / 3} v_{m}
$$

where $h$ is the Plank's constant, $k_{B}$ the Boltzmann's constant and $V_{a}$ the atomic volume. The average sound velocity in the polycrystalline material is given by $[38,39]$ :

$$
v_{m}=\left[\frac{1}{3}\left(\frac{2}{v_{t}^{3}}+\frac{1}{v_{l}^{3}}\right)\right]^{-1 / 3}
$$

where $v_{l}$ and $v_{t}$ are the longitudinal and transverse sound velocity obtained using the shear modulus $G$ and the bulk modulus $B$ from Navier's equation $[38,40]$ :

$$
\mathrm{v}_{l}=\left(\frac{3 B+4 G}{3 \rho}\right)^{1 / 2} \text { and } n u_{t}=\left(\frac{G}{\rho}\right)^{1 / 2}
$$

where $\rho$ is the density of the considered compound. The calculated sound velocity and Debye temperature as well as the density for $\mathrm{Nb}_{4} \mathrm{AlC}_{3}$ are given in Table 5. Unfortunately, as far as we know, there are no data available in the literature on these properties for this compound. Future experimental work will test our calculated results.

\section{CONCLUSIONS}

Employing PP-PW approach based on density functional theory, within the local density approximation, we studied the structural, electronic and elastic properties of $\mathrm{Nb}_{4} \mathrm{AlC}_{3}$ compound. A summary of our results follows.

1. The pressure dependence of the relative lattice parameters $\left(a / a_{0}, c / c_{0}\right)$ and internal structural parameters are calculated. These results should be useful for future work on this compound.

2. This material is found to be conducting.

3. $\mathrm{Nb}_{4} \mathrm{AlC}_{3}$ is stable compound and its bonding is driven by $\mathrm{Nb} d-\mathrm{Al} \mathrm{p}$ and $\mathrm{Nb} d-\mathrm{C} p$ hybridizations. The $\mathrm{Nb}$ $d$-C $p$ bond is stiffer than $\mathrm{M} d$-Al bond.

4. A numerical first-principles calculation of the elastic constants was used to predict $C_{11}, \mathrm{C}_{12}, \mathrm{C}_{13}, \mathrm{C}_{33}, \mathrm{C}_{44}$ and $C_{66}$.

5. We calculated the shear modulus $G$, Young's modulus $E$, and Poisson's ratio $v$, for ideal polycrystalline Nb4AlC3 aggregate.

6. We derived the sound velocity and the Debye temperature for $\mathrm{Nb} 4 \mathrm{AlC} 3$ compound.
[1] M.W. Barsoum, Prog. Solid State Chem. 28, 201 (2000) and references therein.

[2] P. Finkel, M.W. Barsoum, and T. El-Raghy, J. Appl. Phys. 87, 1701 (2000).
[3] M.W. Barsoum, and T. El-Raghy, J. Am. Ceram. Soc. 79, 1953 (1996).

[4] M.W. Barsoum, D. Brodkin, and T. El-Raghy, Scripta Metall. Matter. 36, 535 (1997). 
[5] M.W. Barsoum, M. Ali, and T. El-Raghy, Metall. Mater. Trans. A 31, 1857 (2000).

[6] M.W. Barsoum, and T. El-Raghy, Am. Scientists. 89, 336 (2001).

[7] M.W. Barsoum, L. Farber, T. El-Raghy, and I. Levin, Metall. Mater. Trans. A 30, 1727 (1999).

[8] M. W. Barsoum, and M. Radovic, Mechanical properties of the MAX phases, Encyclopedia of Materials: Science and Technology, Elsevier, Amsterdam, 2004.

[9] M.W. Barsoum, Physical properties of the MAX phases, Encyclopedia of Materials: Science and Technology, Elsevier, Amsterdam, 2006.

[10] J.H. Hettinger, S.E. Lofland, P. Finkel, T. Meehan, J. Palma, K. Harrel, S. Gupta, A. Ganguly, T. El-Raghy, and M.W. Barsoum, Phys. Rev. B 72, 115120 (2OO5).

[11] B. Manoun, F.X. Zhang, S.K. Saxena, T. El-Raghy, and M.W. Barsoum, J. Phys. Chem. Solids 67, 2091 (2006).

[12] M.K. Drulis, H. Drulis, A. E. Hackemer, A. Ganguly, T. ElRaghy, and M. W. Barsoum, J. Alloys Comp. 433, 59 (2007).

[13] B. Manoun, S. K. Sexena, M. W. Barsoum, Appl. Phys. Lett 86, 101906 (2005).

[14] B. Manoun, S. K. Sexena, T. El-Raghy, M. W. Barsoum, Appl. Phys. Lett 88, 201902 (2006).

[15] M. Magnuson, M. Mattesini, O. Wilhelmsson, J. Emmerlich, J.-P. Palmquist, S. Li, R. Ahuja, L. Hultman, O. Erikson, U. Jansson, Phys. Rev. B 74, 205102 (2006).

[16] C.F. Hu, F.g. Li, J. Zhang, J. Wang, J.Y. Wang, Y.C. Zhou, Scripta Mater. 57, 893 (2007).

[17] C.J. Rawn, M.W. Barsoum, T. El-raghy, A. Procipio, C.M. Hoffman, C.R. Hubbard, Mat. Res. Bull. 35, 1786 (2000)

[18] P. Hohenberg, and W. Kohn: Phys. Rev. 136, B864 (1964).

[19] W. Kohn, and L.J. Sham, Phys. Rev. 140, A1133 (1965).

[20] M.D. Segall, P.J.D. Lindan, M.J. Probert, C.J. Pickard, P.J. Hasnip, S.J. Clark, and M.C. Payne, J. Phys.: Condens. Matter 14, 2717 (2002).
[21] D.M. Ceperly, and B.J. Alder, Phys. Rev. Lett. 45, 566 (1980).

[22] J.P. Perdew, and A. Zunger, Phys. Rev. B 23, 5048 (1981).

[23] D. Vanderbilt, Phys. Rev. B 41 (1990) 7892.

[24] H.J. Monkhorst, and J. D. Pack, Phys. Rev. B13, 5188 (1976).

[25] F. Birch: J.J. Geophys. Res. 83, 1257 (1978).

[26] C.D. Gelatt Jr., A.R. Williams, V.L. Moruzzi, Phys. Rev. B 27, 2005 (1983).

[27] I. Salama, T. El-Raghy, and M.W. Barsoum, J. Alloys Compd. 347, 271 (2002).

[28] L. Lpez-de-la-Torrea, B. Winklerb, J. Schreuerb, K. Knorrc, M. Avalos-Borjad, Solid State Comm. 134, 245 (2005).

[29] N.W. Ashcroft, and N. D. Mermin, Solid State Physics, Saunders College (Philadelphia, 1976).

[30] J. F. Nye, Physical Properties of Crystals, Clarendon (Oxford, 1957).

[31] V. Milman, and M.C. Warren, J. Phys.: Condens. Matter 13, 241 (2001)

[32] V. Milman, and M.C. Warren, J. Phys.: Condens. Matter 13, 5585 (2001).

[33] V. Milman, B. Winkler, and M. I. J. Probert, J. Phys.: Condens. Matter 17, 2233 (2005).

[34] M.J. Mehl, B.M. Barry, and D.A. Papaconstantopoulos, Intermetallic Compounds: Principle and Practice, Volume I: Principles, J.H. Westbrook and R.L. Fleischeir, eds., John Wiley and Sons (London, 1995), Ch. 9, p. 195-210.

[35] A. Reuss, and Z. Angew, Math. Mech. 8, 55 (1929).

[36] W. Voigt, Lehrbush der Kristallphysik, Taubner, (Leipzig, 1928).

[37] R. Hill, Proc. Phys. Soc. London A 65, 349 (1952).

[38] Z. Sun, D. Music, R. Ahuja, S. Li, and J.M. Schneider, Phys. Rev. B 70, 508 (2004)

[39] O.L. Anderson, J. Phys. Chem. Solids 24, 909 (1963).

[40] E. Schreiber, O.L. Anderson, and N. Soga, Elastic Constants and Their Measurements, McGraw-Hill, (New York, 1973). 